Sistemas de pensiones y perspectivas de la seguridad social

\author{
Roberto Ham Chande*
}

Al entrar al siglo XXI.México se encuentra en una etapa de desarrollo intermedio, experimentando profundas transformaciones sociales, económicas, políticas y demográficas. Los cambios demográficos muestran un rápido envejecimiento de su población aunado a insuficiencia financiera y déficit actuariales en los sistemas de seguridad social. Asi, un reto a enfrentar, son los grandes requerimientos por pensiones de retiro. El sistema de se guridad social está compuesto por varias instituciones que cubren grupos diferentes de trabajadores: asalariados de compañías privadas, del servicio público y de empresas descentralizadas. Más de la mitad de la población económicamente activa queda del todo excluida; éstos son los trabajadores del campo y el sector informal de menores recursos. Apenas $24 \%$ de la población mayor de 60 años tiene alguin tipo de pensión de retiro, y en su mayoría los estipendios son raquíticos. Los institutos de seguridad social presentan deficiencias producto de los problemas económicos y sociales del país. Bajo distintos escenarios de proyección, aparecen como inevitables grandes desbalances en dos cocientes importantes: entre trabajadores activos y pensionados, entre costos de las jubilaciones $e$ ingresos por salarios. Estas perspectivas obligan a pensar en modificaciones. El principal cambio se ha hecho en el IMSS, el cual cubre a los asalariados urbanos del sector privado. Se ha pasado del sistema de beneficios definidos al de contribuciones definidas. Este sistema no logrará un porcentaje adecuado de reemplazo del salario y las pensiones futuras estarán en su mayoria en el nivel minimo garantizado. Este nivel de garantía corre a cargo de las finanzas públicas, lo cual aunado a los costos de transición significan una gran carga tanto para los afiliados a la seguridad social como para los no afiliados. En términos de activación económica se puede asegurar que con el ahorro interno de la seguridad social, la economía del país no está adaptada para convertir esos recursos en inversión productiva. El problema merece atención política, económica y social con visión a largo plazo.

\title{
Los propósitos de la seguridad social
}

Históricamente, los primeros antecedentes de la seguridad social se dan al final del siglo XIX, principalmente en sus connotados orígenes durante el gobierno alemán del canciller Bismarck, para conformarse como un producto relevante de las transformaciones sociales $\mathrm{y}$ económicas del siglo $\mathrm{xx}$, incluyendo la participación que ha tenido en el desarrollo de México. En una definición resumida, la seguridad social es "la protección que la sociedad otorga contra las contingencias económicas y sociales derivadas de la pérdida de ingresos Norte.

* Profesor-investigador de El Colegio de México y El Colegio de la Frontera 
a consecuencia de enfermedades, maternidad, riesgos del trabajo, invalidez, vejez y muerte, incluyendo la asistencia médica". (OIT, 1984).

Esta definición tiene como base fundamental y razón de ser la equidad y justicia que la seguridad social debe procurar mediante los principios de la solidaridad entre clases sociales y entre generaciones. Se trata de un mecanismo legislado, pactado y obligado de redistribución de la riqueza, de los pudientes hacia los necesitados, de los sanos hacia los enfermos, de los jóvenes a los viejos, de los ocupados a los desempleados. Con estas miras se han creado las distintas instituciones de seguridad social que administran la transferencia de recursos para otorgar servicios y beneficios en protección de la población ante los riesgos señalados. Dentro de estas contingencias y beneficios se incluyen las pensiones ante las pérdidas de la capacidad para el trabajo debidas a los riesgos laborales, la vejez, la enfermedad o la invalidez, asi como a la ausencia del sostén de la familia en la forma de rentas de viudez, orfandad o ascendencia.

Como parte de los cambios sociales y económicos, la seguridad social también se está transformando y responde a las teorías y demandas de cada modelo e intento de desarrollo. Como tal, ha sido particularmente afectada en un fin de siglo que busca liberar mercados y globalizar actividades económicas (Arancibia y Ramírez, 1999). En este artículo se presenta un análisis de las condiciones de la seguridad social en México, enfocado en los sistemas de pensiones y sus prospectivas para las primeras décadas del siglo XXI. ${ }^{1} \mathrm{Se}$ hace una glosa general no sólo sobre las pensiones por jubilación, sino que se incluyen también las de viudez, invalidez, riesgos del trabajo, orfandad y ascendencia. Sin embargo, aunque las pensiones de jubilación corresponden a $39 \%$ de los casos, son las que tienen mayor peso económico.

Las pensiones en el proceso social, económico y político de México

Con algunas primeras manifestaciones desde el porfiriato, la seguridad social se inicia ya en cierta forma con la creación en 1925 de la

\footnotetext{
${ }^{1} \mathrm{El}$ autor agradece los invaluables comentarios y apoyo de J. L. Salas Lizaur y C. Soto Pérez, quienes como actuarios y expertos en seguridad social han brindado generosa ayuda en la elaboración de este artículo.
} 
Dirección de Pensiones Civiles y de Retiro, que en 1959 se transformaría en el actual Instituto de Seguridad y Servicios Sociales de los Trabajadores del Estado (ISSSTE). Los empleados dedicados a actividades relacionadas con el gobierno, las instituciones con presupuesto gubernamental y los grupos policiacos, cuentan con programas de seguridad social administrados por diversas organizaciones. La mayor de estas instituciones es el ISSSTE, el cual agrupa a la burocracia del gobierno federal, a los maestros adscritos a la Secretaría de Educación Pública y a otras organizaciones de carácter público. De modo semejante, en casi todos los estados de la República existe un organismo propio para atender la seguridad social de los empleados estatales y municipales, la policía local y los maestros estatales. ${ }^{2}$ Parte de la existencia de estas instituciones ha venido de la necesidad de conceder beneficios suficientes a los servidores públicos y a las fuerzas armadas para sostener el equilibrio social y político del país.

La consolidación de la seguridad social en México llega en 1943 con el establecimiento del Instituto Mexicano del Seguro Social (IMSS), la más grande de las organizaciones de seguridad social del país. Bajo las tónicas del Estado Benefactor, esta institución de seguridad social se construyó pensando en el modelo económico que miraba al desarrollo mediante la industrialización y sustitución de importaciones. Por medio del IMSS se otorgaban condiciones mínimas de protección a los empleados y a la clase obrera asalariada al servicio de las empresas privadas para garantizar el esfuerzo industrial (IMSS, 1985). El establecimiento del Instituto permitió que las empresas, grandes, medianas o pequeñas, cumplieran con los preceptos de la Ley Federal del Trabajo en materia de protección laboral y seguridad social.

A partir de la pacificación del país, luego del movimiento revolucionario y sus secuelas sociales y económicas, el aparato político y de gobierno creó una serie de empresas calificadas como "descentralizadas", dedicadas a actividades de gran importancia económica, social y estratégica, como son la explotación petrolera, la generación y distribución de electricidad, el manejo de la banca central y de desarrollo,

\footnotetext{
${ }^{2}$ Estos institutos estatales se crearon siguiendo el modelo del ISsSTE, lo cual se refleja en sus nombres y siglas, como son los ejemplos del Instituto de Seguridad Social de los Trabajadores del Estado de Sinaloa en corto conocido como Isstesin, y así se tienen el Isstecali para Baja California, el Issteson en Sonora, el Isstech en Chiapas y de modo parecido en casi todas las demás entidades.
} 
y el funcionamiento de los ferrocarriles. ${ }^{3}$ Dentro de este sector también se incluyó a los empleados del IMSS. ${ }^{4}$ Estas empresas en gran parte fueron expresión del nacionalismo social y económico (cuya máxima manifestación fue la expropiación de la industria petrolera), junto con la necesidad de garantizar desde el poder público el abasto y servicio en áreas vitales, además de asegurar su respaldo político y electoral. Sin embargo, la relevancia y posición de estas empresas otorgó a sus sindicatos una gran capacidad política, negociadora y de presión, lo cual les permitió obtener para sus agremiados los mejores términos en sus contratos colectivos de trabajo, que con frecuencia son concesiones de privilegio (Mesa-Lago, 1986), incluyendo las prestaciones de seguridad social y los beneficios de pensión y de retiro (Valencia, 1999a). El ejército, la marina y la fuerza aérea cuentan con su propio sistema, administrado por el Instituto de Seguridad Social de las Fuerzas Armadas (ISSFAM).

Se pueden distinguir las diferencias existentes entre las distintas organizaciones que componen al sistema de seguridad social por medio de la siguiente descripción resumida de los esquemas de pensiones y sus comparaciones:

1) Para los asegurados del IMSS las jubilaciones se otorgan después de los 65 años de edad, o de 60 en caso de cesantía; el monto de la pensión es un porcentaje del salario promedio de los últimos cinco años que depende del tiempo de cotización. Debido principalmente a los periodos de alta inflación, las pensiones se han reducido tan rápidamente en términos reales que en su mayoría han quedado en el mínimo legal; en el caso de las jubilaciones este límite equivale a un salario mínimo. A su vez, las otras pensiones como las de invalidez, viudez y orfandad tienen montos aun menores. El IMSS ha realizado una reforma que transforma las jubilaciones en un sistema de ahorro individual manejado en las finanzas privadas. Estas modificaciones son obligatorias para los nuevos ingresantes; los jubilados existentes

\footnotetext{
${ }^{3}$ Estos organismos son principalmente Petróleos Mexicanos, Comisión Federal de Electricidad, Luz y Fuerza del Centro, Ferronales, Banobras, Banco de Mexico, Banrural, Nacional Financiera, Banco Nacional de Comercio Exterior, IMss (como empleador).

${ }^{4}$ En cuanto a la seguridad social el IMSs tiene un doble atributo. En su parte primordial se trata de un organismo asegurador al cual se afilian principalmente los trabajadores asalariados urbanos al servicio de las empresas privadas. Por otra parte, tiene sus propios empleados, principalmente burocracia y servicio médico, a los cuales concede beneficios de seguridad social en su calidad de patrón.
} 
se rigen por las condiciones de la ley anterior, mientras que los trabajadores que se afiliaron al IMSS antes de las modificaciones tendrán la opción de jubilarse bajo las nuevas reglas o de acuerdo con la ley anterior (DOF, 1995).

2) En el ISSSTE y en la generalidad de los institutos estatales la jubilación se alcanza desde los 55 años de edad. Su cuantía es un porcentaje del promedio de los salarios del último año, que va de $50 \%$ cuando se tienen 15 años de cotización hasta $100 \%$ cuando se tienen 30 años cotizados. De hecho, cuando se reúnen 30 años de cotización en los hombres y 28 en las mujeres la jubilación se concede sin mínimo de edad. A pesar de estas mejores condiciones y debido a la inflación, las pensiones de jubilación en el ISSSTE tienen un monto promedio de 1.5 veces el salario mínimo (ISSSTE, 1997). Existen variaciones en las legislaciones y los beneficios que se conceden en los distintos sistemas estatales de seguridad social. La mayoría de estos institutos tiene mejores prestaciones que el ISSSTE y son pocos los que adoptan el esquema mínimo de dicho instituto ( $\mathrm{Fa}$ rell, 1998).

3) En las instituciones descentralizadas las condiciones y beneficios por pensiones son contrastantes. Las jubilaciones se igualan al último salario; se dice que son dinámicas porque se actualizan en sus montos en las mismas cantidades en que se revalorizan los salarios del personal en activo; como parte de la jubilación se conceden vacaciones pagadas y aguinaldos de fin de año; en varias de estas instituciones bastan antigüedades de 25 o 27 años para obtener los beneficios completos. De igual manera son privilegiadas las condiciones de las pensiones por otras causas diferentes al retiro (Valencia, 1999a).

\section{Asegurados y pensionados al cambio de siglo}

En el año 2000 se estima para México una población de 99 millones y medio, de los cuales 35 millones forman parte de la población económicamente activa ( $\mathrm{PEA}$ ). Mediante las estadísticas de población y los informes estadísticos de algunas de las principales instituciones de seguridad social, se ha construido el cuadro 1, el cual estima el número de asegurados y pensionados, el número de pensionados por cada 100 activos y cuánto representa el monto de las pensiones sobre el total de los salarios, todas estas variables distribuidas por tipo de institución. 
CUADRO 1

PEA asegurada, pensionados, pensionados por cada 100 asegurados, pensiones/salarios. México, 2000

\begin{tabular}{lcccc}
\hline & $\begin{array}{c}\text { PEA } \\
\text { asegurada }\end{array}$ & Pensionados & $\begin{array}{c}\text { Pensionados } \\
x \text { 100 activos }\end{array}$ & $\begin{array}{c}\text { Pensiones/ } \\
\text { salarios }\end{array}$ \\
\hline Total & 14874 & 2648 & 19.6 & 12.7 \\
IMSS & 11237 & 1820 & 16.2 & 8.5 \\
ISSSTE & 1995 & 414 & 20.8 & 16.7 \\
Inst. estatales & 950 & 123 & 12.9 & 14.4 \\
Org. descent. & 692 & 291 & 42.1 & 32.8 \\
\hline
\end{tabular}

Fuente: Elaboración propia con datos de: Conapo, 1999; IMSS, 1997; ISSSTE, 1997; Farell, R. M., 1999; Salas, J. L., 1998; Valencia, A., 1999a.

Se recalca que las pensiones a las que se hace alusión en este cuadro y en la mayor parte de este texto se refieren no sólo a las jubilaciones de retiro, sino a todos los tipos de pensiones. En su distribución para el año 2000 se tiene que $39 \%$ son de jubilación, $23 \%$ de viudez, $18 \%$ de invalidez, $10 \%$ por riesgos de trabajo, $8 \%$ de orfandad y $2 \%$ se concede a ascendientes.

Bajo estas estimaciones las 2648000 pensiones que se pagarán en el país representan 19.6 pensionados por cada 100 activos asegurados y su costo equivale a $12.7 \%$ de la masa total de salarios. Estas cantidades y relaciones varían entre las diversas instituciones de seguridad social. En el IMSS son 16.2 pensionados por cada 100 activos, en el ISSSTE 20.8, en las instituciones estatales 12.9 , y sube a 42.1 en los organismos descentralizados. En cuanto a los costos, las bajas pensiones del IMSS representan $8.5 \%$ de los salarios, $16.7 \%$ en el ISSSTE, $14.4 \%$ en las instituciones estatales y llega a $32.8 \%$ en las empresas descentralizadas.

Las metas no alcanzadas de la seguridad social

No se cuestionan los adelantos sociales y económicos que para México significaron las acciones de las distintas instituciones a cargo de la seguridad social, sino que se reconocen los beneficios que lograron en favor de la salud y el bienestar de la población del México moder- 
no, particularmente de las emanadas por el IMSS. Sin embargo, lo que ha sido difícil de admitir es que la seguridad social, según se conformó en nuestro país y también en las dẹmás naciones de América Latina, siempre ha estado lejos de cumplir plenamente con sus objetivos sociales y finalmente parece no encontrar los medios para su permanencia hacia el siglo XXI como mecanismo de solidaridad (Laurell, 1996).

Fijando la atención en el papel que la seguridad social debería cumplir en la protección en cuanto a jubilaciones y los demás tipos de pensiones, no puede soslayarse que:

1) De acuerdo con las estimaciones el porcentaje de la PEA inscrita en algún sistema de seguridad social es sólo $41 \% .{ }^{5}$ Bastante más de la mitad de la población económicamente activa no cuenta con una cobertura de la seguridad social que alguna vez le permita ya sea jubilarse o pensionarse debido a enfermedades e incapacidades, o generar una pensión para sus dependientes. Los carentes de la seguridad social son justo los más débiles e incapaces de generar presiones económicas o políticas en busca de derechos, y principalmente se encuentran entre los trabajadores rurales, los del sector informal y los desempleados. Se destaca que este problema es parte del notorio y eterno descuido de las áreas rurales, que alcanza dimensiones de sacrificio para subsidiar al sector industrial, al aparato de gobierno y ahora también a las exportaciones.

2) En gran parte como consecuencia del punto anterior, las estimaciones nos dicen que sólo $24 \%$ de la población mayor de 60 años de $e{ }^{6} d^{6}$ recibe algún tipo de pensión y los que no la tienen pertenecen a los grupos marginados y vulnerables. Por su bajo monto, este porcentaje ejemplifica la desprotección de la población en edad avanzada en cuanto a cobertura por pensiones. Se incluyen todas las instituciones de seguridad social y no sólo las jubilaciones sino también las pensiones por viudez, por riesgos de trabajo en edad avanzada y para los ascendientes.

${ }^{5}$ Ésta es la cifra que se obtiene partiendo de los registros de afiliación de las instituciones de seguridad social y es la que se utiliza por coherencia con las fuentes de datos utilizadas. Sin embargo, las estimaciones que resultan de censos y encuestas dan cifras menores.

${ }^{6}$ Ese corte de edad considera que en el IMSs una gran parte de las jubilaciones se toman desde los 60 años bajo el beneficio de "cesantía en edad avanzada". Asimismo, como se ha mencionado, en las otras instituciones de seguridad social hay una proporción significativa que se jubila antes de esa edad. 
3) La cuantía de la mayoría de las pensiones no es suficiente para cubrir las necesidades más mínimas. Estas deficiencias se ligan a la inflación -que minimiza los montos iniciales-y a la insuficiencia de los salarios-sobre todo en las últimas dos décadas- que sirven como base de cotización y también para el cálculo de las pensiones.

4) Debido a la falta de universalidad de la cobertura y a las diferencias entre los sistemas de seguridad social, en términos redistributivos la seguridad social es un esquema regresivo en favor de los más privilegiados y en gran parte a costa de los más necesitados (Guhan, 1994). Esta deficiencia no es exclusiva de México; también es notoria en otros países en desarrollo, particularmente los de América Latina. Se debe a la utilización de recursos públicos en las contribuciones y subsidios del Estado a las diferentes instituciones de seguridad social, y a la asimilación de las cuotas de empleados y patrones a costos de productos y servicios, que a su vez repercuten en incrementos de precios y limitaciones al consumo.

\section{Deficiencias financieras y recomposición de la seguridad social}

Sólo hasta recientemente se ha escrito, discutido y explicado en foros abiertos las razones por las cuales la seguridad social en México nunca llegó a cumplir con su cometido de ser el mecanismo solidario y redistribuidor de la riqueza, que debería proteger a todos los mexicanos y hacerlo con beneficios adecuados. Con el afán de buscar una recomposición de la seguridad social, interesa abordar lo relativo a las pensiones en general pero particularizando en las pensiones de retiro, en sus posibilidades, y también en sus fallas.

De modo relevante, también cabe admitir que no han sido las deficiencias de la seguridad social las que han desencadenado la necesidad de reformarla. Lo que ha atraído la atención política y económica -tanto en México como en el resto de América Latina- sobre el futuro de la seguridad social ha sido su proximidad al colapso económico ante la insuficiencia financiera y el escalamiento del déficit actuarial, que hacen evidente la dificultad de cumplir con los compromisos pactados en leyes, acuerdos y contratos colectivos de trabajo.

Asimismo, debe considerarse que en sus virtudes y defectos así como en sus problemas financieros, la seguridad social y sus instituciones son parte del desempeño económico, social y político del país 
en general (Salas, 1998). Gran parte de su crítica falta de recursos ha sido consecuencia de las dificultades económicas, la falta de empleos, la baja real de los salarios, y de la creciente informalidad del trabajo, factores que disminuyeron la afiliación y contribución a la seguridad social. De modo paralelo, estas mismas crisis crearon mayor demanda y costo por los servicios médicos en todas las instituciones de seguridad social (IMSS, 1995).

Como se menciona en diagnósticos ampliamente aplicados al IMSS, en argumentos semejantes a los que ya se manejan para el ISSSTE y las demás instituciones de seguridad social, los factores que provocaron las dificultades financieras y la limitación de alcances incluyen: bases biométricas y actuariales rebasadas por la dinámica demográfica, concesión de beneficios sin respaldo financiero, deficiencias administrativas, evasión de aportaciones, desviación de recursos y distracción de reservas ${ }^{7}$ (IMSS, 1997). Pero poco se menciona acerca del escaso control en el uso de recursos, en la concesión de beneficios sin previsiones actuariales, y del atentado a la solidaridad al concederse beneficios de privilegio a grupos especiales atendiendo razones de presión sindical o política, en una desafortunada situación generalizada en toda América Latina.

\section{Desbalance entre el financiamiento y el costo de las pensiones}

La visión más severa sobre los problemas de las pensiones y la seguridad social no surge de la situación actual de déficit presente, sino de lo que se espera de acuerdo con las proyecciones de costos. Una primera consideración es darse cuenta que los cálculos actuariales que sirvieron como base técnica en la fundación y arranque de las instituciones de seguridad social se realizaron con parámetros demográficos que difirieron grandemente de la dinámica que posteriormente se observó. En el caso de las jubilaciones se esperaba que menos supervivientes llegaran a las edades de retiro y que su esperanza de vida fuera menor una vez llegada la:jubilación. Al inaugurarse el IMSS en 1943 sus bases actuariales se calcularon con la tabla de mortalidad

${ }^{7}$ Habría que conceder que la ampliación y subsidio de los servicios médicos a costa de las reservas técnicas de las pensiones ha sido una verdadera inversión que si no generó rendimientos financieros, sí ha sido elemento de bienestar de los asegurados y de la población en general. 
Hunter Tropical, que se suponía sobrestimaba la esperanza de vida a edad alcanzada de 65 años, considerándola en ocho años (Soto, 1992a). Sin embargo, desde esa época ya las esperanzas de vida tenían valores por encima de ocho y ahora sobrepasan los 15 años y muestran tendencias crecientes hacia el futuro. Desde el balance actuarial de 1960 se advirtió un déficit actuarial, ${ }^{8}$ el cual se incrementó en los subsecuentes balances hasta el último, realizado en $1969,{ }^{9}$ pero estas advertencias fueron ignoradas por las administraciones del momento por considerarlas políticamente improcedentes (Soto, 1992b).

De esta manera, independientemente de las fallas administrativas, las aportaciones que se cubrieron resultaron absolutamente insuficientes para pagar los beneficios que se concedían. Una ilustración numérica simplificada se ofrece en la gráfica 1 , en la cual se supone que un individuo se inscribe como asegurado del IMSs a los 23 años de edad, trabaja durante 37 años ininterrumpidamente, su salario crece $1.0 \%$ anualmente en términos reales, sus aportaciones son de $6.0 \%$ de su salario de acuerdo con la Ley del IMSS anteriormente vigente, estas aportaciones no sufren mermas y producen un rendimiento de $3.5 \%$, también por encima de inflación; llega a la edad de 60 años cuando se jubila por cesantía en edad avanzada, recibiendo $70 \%$ de su último salario, beneficio otorgado por la legislación anterior, para permanecer como pensionado durante 22 años, que en 1995 era la esperanza de vida a la edad de 60 años.

Las barras en el lado izquierdo de la gráfica 1 representan el valor presente, al momento de la jubilación, de las aportaciones correspondientes a la prima para la pensión por jubilación. Éstas suman el equivalente a 3.6 años del último salario, lo cual es entonces lo ingresado al IMSS en este caso. En el lado derecho de la edad al retiro las barras representan el valor presente de la pensión de retiro, las que tienen un valor de 12.0 años de salario, cantidad que es la obligación financiera a la que el IMSS se obliga por esta jubilación.

Es claro que bajo cualquier circunstancia el IMSS, incluso con la administración perfecta, llegaría al desfinanciamiento y al desbalance actuarial. Este mismo tipo de ejercicio lleva a conclusiones no sólo se-

\footnotetext{
${ }^{8}$ Un déficit actuarial es la diferencia negativa entre los valores presentes de los pagos esperados por beneficios y los cobros por contribuciones.

${ }^{9}$ Luego de ese último balance actuarial, la planeación financiera del IMss se basó en proyecciones.
} 


\section{GRÁFICA 1}

Valor presente de aportaciones y pensión de acuerdo con la anterior Ley del Seguro Social

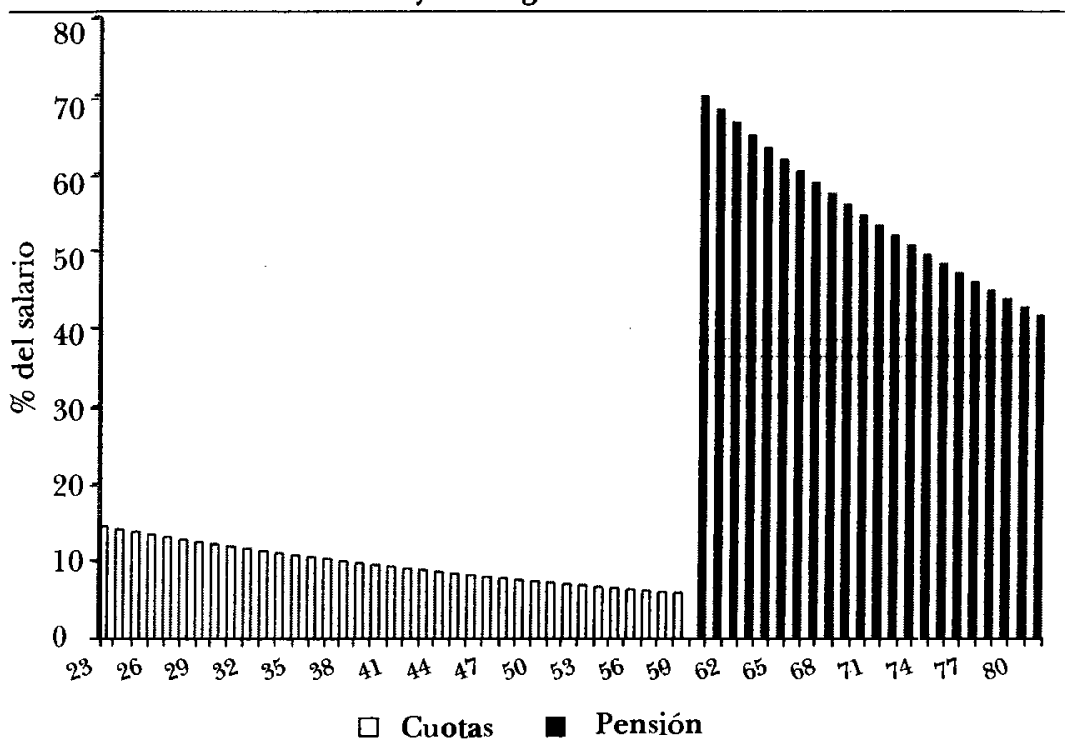

Supuestos: prima de aportación de $6.0 \%$; tasa real de interés de $3.5 \%$; tasa de crecimiento de salario de $1.0 \%$.

Fuente: Elaboración propia.

mejantes, sino incluso extremas, en las demás instituciones y sistemas de pensiones, particularmente cuando se trata de los esquemas privilegiados de los organismos descentralizados.

Prospectivas sobre los costos del sistema de pensiones

Si se considerara al colectivo de asegurados y pensionados de todas las instituciones de seguridad social y en los diferentes tipos de pensiones, pueden construirse estimaciones como las que se presentan en las siguientes tres gráficas, donde se ilustran las proyecciones de los conceptos mostrados en el cuadro 1 para las décadas desde el año 2000 hasta el $2050 .{ }^{10} \mathrm{La}$ intención es estimar el desarrollo esperado

${ }^{10}$ Las gráficas 2, 3 y 4 son ilustración visual de las proyecciones de los conceptos utilizados en el cuadro 1. 
durante la primera mitad del siglo XXI del número absoluto de pensiones, del número de pensiones por cada 100 activos y del monto que representan estas pensiones como porcentaje de los salarios, tanto para el total como en su distribución entre los distintos tipos de instituciones de seguridad social.

En este punto se hace necesaria una advertencia metodológica. Un ejercicio de prospectiva como el que aquí se propone debería partir de una gran valuación demográfica, económica, actuarial y social del sistema de seguridad social en su conjunto y de las instituciones que lo componen. Sin embargo, actualmente no existe un acervo de información y de evaluación que por una parte sea de acceso público y que por otro lado tenga suficiente flexibilidad para admitir distintas hipótesis de proyección y la construcción de escenarios. El ejercicio de prospectiva que aquí se presenta parte de información estadística y actuarial general que por una parte está agregada, pero también es fragmentada y limitada. Parte de la guía de este ejercicio se basa en las valuaciones actuariales de algunas instituciones ${ }^{11}$ junto con las proyecciones resultantes. Otro insumo determinante viene de lo que sucede en otros países que sí cuentan con prospectivas y datos publicados. Si en naciones de todos los niveles de desarrollo los sistemas de seguridad social prevén serias dificultades a futuro, lo seguro es que en México suceda lo mismo. Esto ha permitido elaborar con cierto grado de confianza las hipótesis generales de desarrollo y cobertura del sistema de seguridad social, traducidas en las prospectivas que se analizan. Finalmente, el ejercicio se ha beneficiado de la discusión con colegas actuarios, quienes en su experiencia y percepción concuerdan en que lo que aquí se presenta está en el lado conservador de las estimaciones.

Una vez aceptado este ejercicio y partiendo de las proyecciones demográficas del Conapo, se muestra que en los primeros 50 años del siglo XXI la población de México se multiplicará por 1.3. Con base en la participación como PEA de los grupos en las edades intermedias y en los supuestos de cobertura de la seguridad social, en la gráfica 2 se expresa que la población pensionada se multiplicará por 4.2, pasando de 2648000 a 11200000 , y la mayor parte de los cuales estará a cargo del IMSS.

\footnotetext{
11 Valuaciones en las que se ha intervenido profesionalmente o a las que se ha tenido acceso como consulta.
} 
GRÁFICA 2

Proyección del número de pensionados (miles) por institución de afiliación. México, 2000-2050

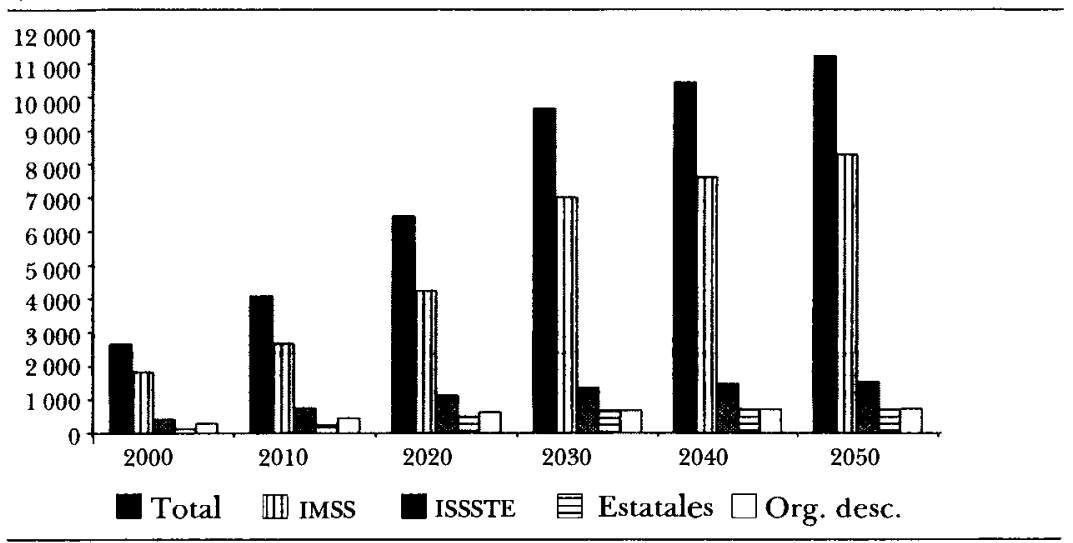

Fuente: Elaboración propia con las mismas fuentes del cuadro 1.

Ciertamente se trata de un fenómeno con grandes repercusiones y parte de las implicaciones de estas dinámicas se dejan ver mejor en las otras dos gráficas. La gráfica 3 expresa el número de pensionados por cada 100 trabajadores activos. Para el total es de 19.6 en el año 2000 y se remontará a 55.5 en 2050 . En la gráfica se nota que estos índices de dependencia son en alto grado determinados por el IMSS, en razón de su tamaño relativo. Luego se nota que en las otras instituciones estos índices están por encima de los promedios del total, pero se destaca lo alto que son en los organismos descentralizados. Ya en el año 2000 será de 42.1 y superior a lo que la generalidad tendrá hasta 2050, año en que será superior a 108 en este tipo de empresas. ${ }^{12}$

Finalmente, respecto a los costos, su comportamiento será también creciente y diferencial. La carga de las pensiones se expresa como el valor de las pensiones en relación con el conjunto de los salarios y se ilustra en la gráfica 4 . En el total este costo es de $12.7 \%$ de los salarios en el año 2000 y llega a $35.0 \%$ en 2050 , lo cual en primer lugar da idea de la carrera ascendente del costo de las pensiones, pero

${ }^{12}$ En este ejercicio de proyección, las empresas descentralizadas aparecen como las de mayor incertidumbre ante las tendencias observadas hacia su privatización y permanencia. 


\section{GRÁFICA 3}

Proyección del número de pensionados por cada 100 activos, por institución de afiliación. México, 2000-2050

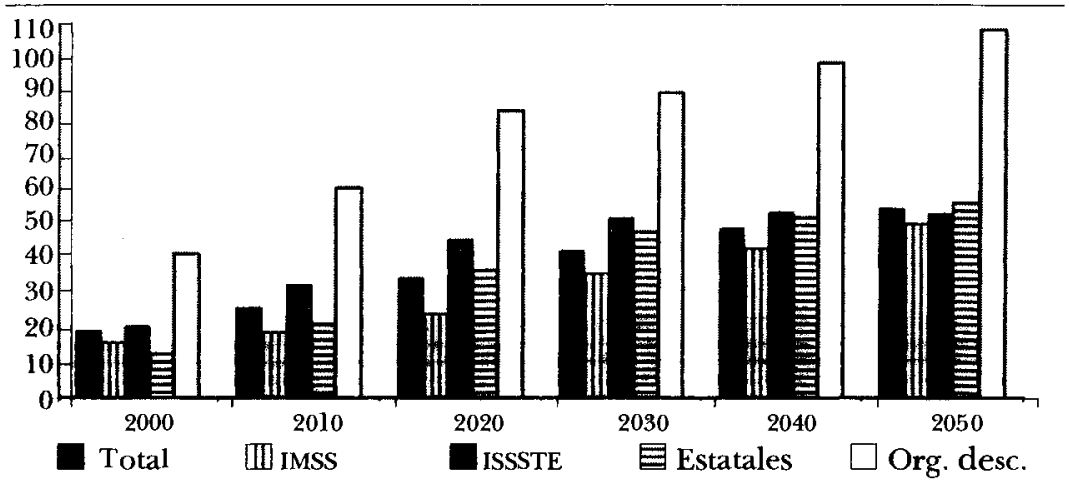

Fuente: Elaboración propia con las mismas fuentes de datos del cuadro 1.

una característica mas notable es la gran variabilidad entre las instituciones respecto a este concepto. Por lo pronto, ninguno de los tipos de instituciones se acerca al promedio del total.

El IMSS, a pesar de tener un gran peso en términos de los números de asegurados y pensionados, se encuentra muy por abajo de los promedios. Por una parte muestra lo bajo del monto de las pensiones que concede pues su costo relativo es de $8.5 \%$ de los salarios en el 2000 y llega a $26.7 \%$ en 2050 , incluso cuando se han calculado respecto a salarios bajos, pero también interviene la mayor base en términos de estructuras por edad y número de contribuyentes.

Por otra parte, en el ISSSTE se comienza con $16.7 \%$ y se llega a mitad de siglo a $43.5 \%$. En las instituciones estatales es $14.4 \%$ en 2000 y se remonta a $64.0 \%$ en 2050 ; esta mayor rapidez en el costo de estas instituciones respecto al ISSSTE se debe a la variedad de organizaciones que las componen, algunas de las cuales tienen altos niveles de beneficios, parecidos a los de los organismos descentralizados. Y en otra manifestación de las mejores condiciones en la concesión de beneficios en los organismos descentralizados los costos de las pensiones ya son equivalentes a $32.8 \%$ de su nómina y este porciento llegará a 84.5 hacia mitad de siglo.

Cifras, estimaciones y proyecciones como éstas aparecen en muchas otras naciones del mundo, en particular en los países de desarrollo intermedio y gran velocidad en su transición demográfica, parecidas a las condiciones de 


\section{GRÁFICA 4}

Proyección del costo de pensiones en relación con el total de salarios por institución de adscripción. México, 2000-2050

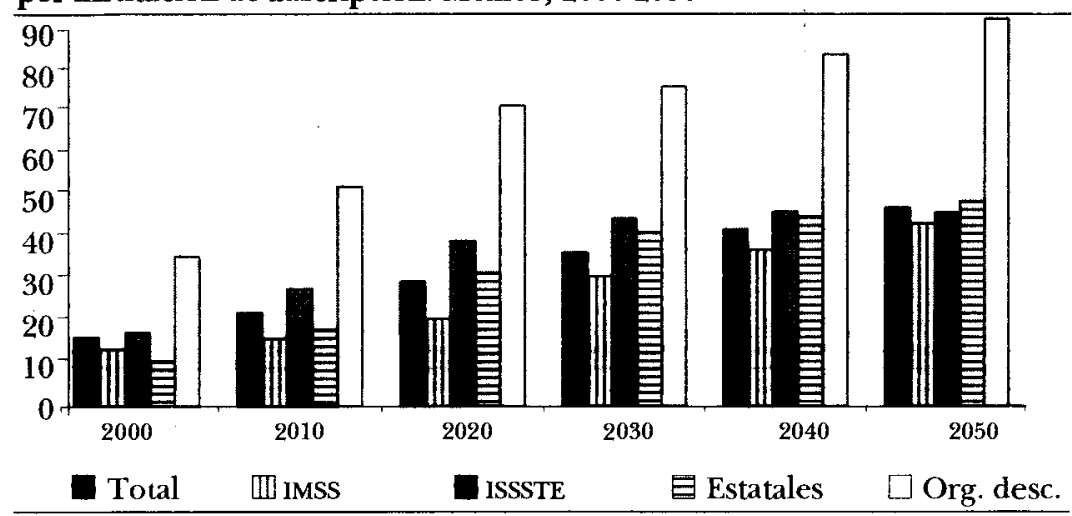

Fuente: Elaboración propia con las mismas fuentes del cuadro 1.

México. La cuestión que imponen es, desde luego, la capacidad para enfrentar los crecientes costos en los niveles de compromiso que se anticipan y plantean la buisqueda de alternativas y soluciones.

\section{Una iniciativa latinoamericana sobre la seguridad social}

En México, al igual que en América Latina en general, las instituciones de seguridad social viven una dinámica en la cual se ha acumulado una población considerable con derecho a pensiones de todo tipo: retiro, invalidez, riesgos de trabajo, viudez, orfandad y ascendencia. Al mismo tiempo que se crea esta gran masa de adquisición de derechos, las condiciones económicas y demográficas del país no permiten un crecimiento en su afiliación ni en el cobro de cuotas que le permitan los recursos para enfrentar obligaciones. Parte de este proceso se atribuye al envejecimien to de su población, cuyas etapas y características están muy ligadas al grado de desarrollo debido a la concomitancia existente con la transición demográfica, pero son más impactantes las condiciones económicas y del mercado de trabajo.

De esta manera y ante la inviabilidad financiera de los sistemas de seguridad social se hace necesario reconsiderar sus propósitos y formas en busca de resolver un problema ya con manifestaciones críti- 
cas. En estos objetivos América Latina se ha mostrado casi como pionera en la reestructuración de los sistemas de pensiones, ${ }^{13}$ transformándolos de un esquema de redistribución bajo los principios solidarios de la seguridad social, en un esquema individual de ahorro obligado.

Ha sido notorio el caso de Chile, como el primer país de América Latina en establecer este tipo de reforma en mayo de 1981. En las últimas décadas del siglo XIX el canciller de hierro en Alemania, Otto von Bismarck, estableció las pensiones de retiro, y en las últimas décadas del siglo Xx Augusto Pinochet, como dictador de hierro en Chile, puso en marcha las reformas de la seguridad social hacia la privatización y la capitalización individual para el retiro (Wallace, 1999), cada uno bajo circunstancias y objetivos distintos. La razón de su gran difusión en América Latina viene de que el nuevo sistema pareció haber actuado con "éxito" durante los primeros 12 años, producto asimismo del avance económico macro logrado por Chile. Ese buen desempeño financiero de los primeros años y las proposiciones de la banca internacional han llevado a otros países a actuar bajo acciones semeijantes. Además de Chile, otros siete países de América Latina han promovido las reformas de la seguridad social en la misma línea de la cu a individual, aunque con variantes en cada uno. Cronológicamente estos países han sido: Perú (junio de 1993); Colombia (abril de 1994); Argentina (julio de 1994); Uruguay (marzo de 1996); Bolivia (mayo de 1997); México (julio de 1997); El Salvador (abril de 1998) (Soto, 1999).

Adicionalmente, otros nueve países han discutido en el terreno político y económico cambios semejantes y de posible implementación: Brasil, Costa Rica, Ecuador, Guatemala, Honduras, Nicaragua, Panamá, Dominicana y Venezuela.

\section{Las reformas de la seguridad social en México}

Ha sido sólo a partir de las urgencias financieras cuando se han buscado soluciones que en el caso de México han tenido dos experiencias principales. Las primeras en el tiempo han sido las refor-

${ }^{13}$ Los sistemas de capitalización individual se han establecido antes en algunos países de Asia, aunque con características diferentes y la principal es que son administradas públicamente. 
mas al funcionamiento del Instituto de Seguridad y Servicios Sociales del Estado de Nuevo León (Isssteleón) ocurrida en 1995, a la que le ha seguido la más notoria reforma del IMss, implantada a mitad de 1997.

En el IMSS el principal cambio ha sido la adopción de un sistema individual para el financiamiento de las pensiones por jubilación futuras. Se establecen cuentas individuales, manejadas en el sector financiero privado por medio de las Administradoras de Fondos para el Retiro (conocidas como Afores), con las cuales eventualmente se comprará una renta vitalicia en una compañía de seguros o se fijarán retiros programados, no contingentes, mediante montos y tiempos definidos. Esencialmente se crea una pensión futura de acuerdo con las posibilidades de ahorro de cada contribuyente, la cual se aleja del principio básico de solidaridad entre generaciones y en tre clases sociales que caracterizaba a la seguridad social. En lugar de ser asegurado se es ahora participante involuntario de una empresa de inversiones (Briseño, 1999).

Cuando se plantearon los cambios se argumentaba, en primer lugar, la necesidad de contar con un sistema que garantizara beneficios dignos y justos después del retiro, con equidad financiera, actuarial y administrativa, transportable de un lugar de trabajo a otro, al cual además se le atribuía la virtud y la tarea de convertirse en un mecanismo creador de ahorro interno y generador de empleos. Un remanente de la seguridad social queda en la garantía de una pensión mínima y semejante a un salario mínimo, si se cumple al menos con un tiempo de cotización equivalente a 24 años. En este caso la diferencia entre lo acumulado en la cuenta individual y el costo de la pensión mínima queda a cargo del erario público.

La importancia obvia de la reforma al IMSS estriba en el tamaño e importancia social y económica de esta institución dentro del contexto nacional. Estas reformas hacia la capitalización individual tienen un antecedente inmediato en la Ley de los Sistemas de Ahorro para el Retiro de 1996. Esta disposición obliga a los empleadores de afiliados al IMSS e ISSSTE a aportar $2.0 \%$ del salario en una cuenta individualizada administrada por el sistema bancario. Con la nueva ley estas cantidades se agregan a la capitalización individual. De igual manera, el $5.0 \%$ de aportación al Infonavit ahora forma parte de la cuenta individual. 
El destino de la solidaridad y la prima media general ${ }^{14}$

Desde el punto de vista económico, en cualquier situación la función del sistema de pensiones es apartar una porción de la producción generada por la población trabajadora para el consumo de los pensionados. Esta premisa es clara y directa en los sistemas de reparto, donde las aportaciones de los activos del momento pagan las pensiones también del momento. Sin embargo, cabe recordar que incluso en el sistema de reparto no se trata de una simple redistribución y solidaridad pura entre generaciones, de los más jôvenes a los más viejos, pues cada generación deja una infraestructura y un legado social y económico para el desarrollo y desempeño de la siguiente.

En los inicios del IMSS y de todas las demás instituciones de seguridad social, los pensionados por jubilaciones no existían; se generaron paulatinamente y a largo plazo. Al mismo tiempo el país entraba en ese periodo histórico de crecimiento económico que hasta se calificó como "milagro mexicano" (Pedrero, 1998). La relación entre pensionados y asegurados era baja, la producción era alta, y en el método financiero y actuarial de prima media general, en lugar de crear las reservas técnicas del sistema de pensiones, estos recursos se tomaron como sobrantes para muchos otros efectos, entre los que destaca la ampliación del sistema y el subsidio al servicio médico. El esquema se convirtió así en uno de reparto, donde en un principio lo que se repartía en forma de pensiones era bastante menos de lo que ingresaba.

La cuestión ahora crucial es que el sistema de reparto era plenamente costeable cuando las exigencias por pensiones eran pocas y las contribuciones a la seguridad social resultaban bastante más que suficientes. Pero esto último es lo que se está revirtiendo en la seguridad social mexicana y en la de todo el mundo. La acumulación de pensiones y pensionados y las transformaciones demográricas y económicas de la población cubierta por la seguridad social generan ahora un creciente déficit financiero y actuarial en magnitudes tales que son imposibles de cubrir.

${ }^{14}$ En teoría, la prima media general sería esa aportación que como porcentaje fijo de los salarios financiaría el sistema a través de la capitalización de la diferencia inicial entre altos ingresos y bajos costos, lo cual permitiría sufragar al sistema cuando los costos sobrepasaran las aportaciones en una perenne estabilidad demográfica y financiera. 
Se crea así una clara necesidad de reformular la estructura y los procedimientos de la seguridad social. Lo que suscita cuestionamientos es la adecuación de las reformas adoptadas y la capitalización individualizada. Sin embargo, no se requiere de mucha teoría para entender que una previsión que otorgue pensiones de vejez mediante cuentas individuales en fondos de inversión realmente funcionaría y cumpliría sus cometidos de protección social con beneficiosijustos otorgados universalmente cuando las aportaciones sean suficientes, exista una economía sana con fortaleza para resistir embates externos, de bajo desempleo, sin población marginada, con capacidad real para el ahorro, donde sus mercados financieros fueran administrados adecuadamente y a salvo de inflación (Uthoff, 1997).

Las escasas expectativas de la capitalización individual

Un efecto deseado por todos es la adecuación financiera de las pensiones, que permita que al final de la vida activa o cuando sobrevenga una enfermedad, se presente una incapacidad, $u$ ocurra la muerte del sostén del hogar, se tengan los recursos para mantener una vida económicamente independiente y en niveles de dignidad. Este anhelo ha sido promesa de la reforma a la seguridad social y se asegura en todos los medios de comunicación. Sin embargo los fondos acumulados en las cuentas individuales tendrán muchas condicionantes antes de conseguir sus objetivos de sustituir al salario durante el final de la vida y el tiempo del retiro. Diversos autores han hecho cálculos actuariales sobre proyecciones de carreras salariales, tasas de interés y montos de anualidades, coincidiendo todos en lo lejos que quedan esas metas de hacerse realidad (Hazas, 1996; Salas, 1998; Partida, 1998). En estas estimaciones, aun bajo perspectivas optimistas sobre tasas reales de interés y con carreras salariales sostenidas y en aumento por más de tres décadas, las cifras calculadas muestran que la renta vitalicia que se alcanzaría como pensión de retiro, comprada en una aseguradora con el dinero acumulado en una cuenta individual, expresa un porcentaje inferior al último salario.

$\mathrm{El}$ nuevo sistema de pensiones de retiro resuelve el desequilibrio entre escasas aportaciones y grandes cobros por pensiones, ${ }^{15}$ desequi-

${ }^{15}$ Aun las magras pensiones mínimas son costosas cuando se ponen en relación con las bajas aportaciones. 
librio que ya se ilustró y comentó en la gráfica 1. Sin embargo, el equilibrio se establece concediendo como pensión exactamente lo poco que se puede comprar. Por otra parte, la insuficiencia de las aportaciones se agrava ante las altas comisiones de administración que requiere un proceso tan costoso como es manejar millones de cuentas individuales en su mayoría cada una de monto pequeño, al mismo tiempo que se sostienen gastos de comercialización y las necesarias utilidades que persigue un sistema privado.

Otros factores que subyacen son los supuestos de una formalidad y estabilidad en el empleo, en una carrera laboral con ascensos reales en el salario. Esto es incierto ante la tendencia de los mercados de trabajo hacia la informalidad, la inestabilidad en el empleo y las experiencias de decrecimiento en el salario real (Forrester, 1996). Otras proyecciones actuariales se han hecho siguiendo la experiencia observada en las Encuestas Nacionales de Empleo Urbano del INEGI ${ }^{16}$ donde se muestra que la densidad de cotización no es de $100 \%$, lo cual reduce notoriamente el porcentaje de sustitución del ingreso (Partida, 1998).

La capitalización individual y la productividad económica

En cuanto a la capitalización individual como ahorro para el retiro, de cualquier forma el consumo de los pensionados también tendrá que venir de la producción y los servicios que ofrezcan los activos en el momento en que se deban satisfacer las necesidades, sólo que en este sistema se intenta contabilizar las aportaciones al aparato productivo del futuro y cobrar de él en razón de esta acumulación y contabilidad individual, con el supuesto adicional de incrementar la productividad de las generaciones futuras a través del ahorro interno que representa la capitalización total y su conversión a inversión productiva promotora de empleos.

De hecho existen muchas condicionantes de carácter económico, financiero y social que deben satisfacerse para que se cumplan las metas económicas y sociales prometidas. Esto lleva a la cuestión del origen y significado económico de las tasas de interés, de la funcionalidad y solidez de los mercados financieros, y de su capacidad para apoyar al sis-

${ }^{16}$ Instituto Nacional de Estadística, Geografia e Informática. 
tema productivo, lo cual todavía no es el caso en las actuales condiciones de México (Sandoval, 1998). Las tasas de interés no deben ser especulativas ni ficticias, apoyadas únicamente por las finanzas públicas, sino que deben ser parte de una economía social e industrialmente productiva. Finalmente, el funcionamiento de la seguridad social, con o sin reformas, necesariamente está basado en la productividad del aparato económico, la solidez del empleo formal, y la fuerza social y laboral de la nación.

Sin embargo, estas virtudes deseadas no parecen funcionar en el sistema de capitalización individual. Ha sido experiencia en la mayoría de los países que los fondos de pensiones se invierten en papeles del gobierno. En el caso de México por el momento la razón técnica viene de que los reglamentos exigen que en los primeros años la inversión se haga en inversiones indexadas, con calificaciones de riesgo mínimo, y sólo es el gobierno quien tiene la autorización para emitir esa clase de instrumentos.

Adicionalmente, las reformas al IMSS dejan una considerable responsabilidad a las finanzas públicas. Una de ellas es el costo de la transición, entendida ésta como el pago de las pensiones fincadas en el sistema anterior y que estarán en vigencia por varias décadas más, hasta la extinción de las cohortes de pensionados que se retiren bajo las normas y condiciones de la ley anterior. Estas generaciones aún se siguen incrementando ante la incorporación de los pensionados que se jubilarán en los próximos años, las que se inclinarán por el antiguo sistema ya que al momento del retiro no habrán acumulado recursos suficientes en una cuenta individual que no habrá estado vigente el tiempo suficiente. ${ }^{17}$

Otro renglón que alguna vez deberá sostenerse por las finanzas públicas es la garantía de las pensiones mínimas. Así varios gastos sumados, como el costo de la transición, las contribuciones del Estado y la garantía de las pensiones mínimas, corren a cargo del gobierno, lo cual en realidad es un costo para todos los contribuyentes y los no contribuyentes, en otra expresión de la regresión negativa dentro del sistema redistributivo. Esta es una incidencia directa y de consideración sobre el grave problema de la desigual distribución del ingreso en México.

${ }^{17}$ A los trabajadores ya afiliados al IMss al momento de las reformas se les permite optar entre el antiguo y el nuevo sistema. 
Algunas consideraciones sobre las prospectivas de la seguridad social

Debe admitirse que ante los problemas nacionales la actividad académica tiene una gran capacidad para diagnosticar y advertir. Sin embargo, esto muy pocas veces va acompañado de actitudes propositivas. Ciertamente que proponer con sentido y realidad en muchas ocasiones es difícil, y que a veces parece imposible, como en el caso del futuro de las pensiones y la seguridad social; empero debe aceptarse la responsabilidad de intentarlo. Tratando de adaptar esta actitud y en lo posible agregar elementos a una discusión no acabada, se presentan las siguientes consideraciones, mezcla de conclusiones, premisas, planteamientos y preguntas acerca de las perspectivas de las pensiones dentro de la seguridad social.

\section{Las áreas críticas}

-El sistema de pensiones y seguridad social en México está compuesto por diversas instituciones, cada una de las cuales cubre a un grupo específico de trabajadores y sus familias. El tipo de beneficios que se concede está en función de la importancia política de los grupos asegurados, de su capacidad de presión (Mesa-Lago, 1986) y del valor que guardan respecto al modelo de desarrollo económico adoptado (Arancibia y Ramírez, 1999). De ahí que, en otra expresión de injusticia, los trabajadores del campo, los del sector informal, los desocupados y los subempleados hayan quedado fuera de la protección.

-La dinámica demográfica, el envejecimiento de la fuerza de trabajo asegurada y la acumulación de riesgos proyecta incrementos en la demanda de beneficios adquiridos y en los costos de las pensiones, que están por encima de toda posibilidad financiera de ser cubiertos mediante las contribuciones que se hacen al sistema. Una respuesta ante este problema ha sido la privatización de las pensiones mediante una capitalización individual, adoptada por algunas instituciones de seguridad social, entre las cuales destaca la llevada a cabo por el IMSS.

-Sin embargo, se ha demostrado que el sistema de pensiones a través de cuentas individuales de capitalización, según se ha adoptado, no logra una sustitución adecuada del ingreso, ni siquiera bajo condiciones óptimas de altas tasas reales de rendimien to y de una prolongada e ininterrumpida vida de trabajo y cotización (Hazas, 
1996; Salas, 1998). Esta situación se agrava cuando se consideran densidades de cotización inferiores a $100 \%$, tal y como muestran las encuestas de empleo (Partida, 1998). En este sentido, el futuro de los mercados laborales en cuanto a sus tendencias hacia la informalidad (Forrester, 1996) disminuye sustancialmente las posibilidades de los esquemas de capitalización individual.

-Actualmente la economía mexicana no tiene las condiciones para convertir al nuevo sistema de seguridad social en un mecanismo de ahorro interno, de inversión productiva y de generación de empleos (Sandoval, 1998); asimismo existen incertidumbres sobre el comportamiento de los mercados financieros para el manejo de los recursos de la capitalización individual (Heller, 1998).

-Con las reformas de la seguridad social se acentúan los mecanismos regresivos de redistribución del ingreso a través de los compromisos por pagar mediante los recursos públicos los costos de las pensiones en transición y las garan tías de las pensiones mínimas.

\section{En busca de propuestas}

-Se debe comenzar aceptando que las pensiones y su futuro son un problema inminente y con gran potencialidad de crisis, demasiado dificil y complejo, el cual no permite una solución simple y directa. La primera premisa de esta aseveración es que las condiciones en las que vienen operando los sistemas de pensiones de beneficio definido y esquemas de reparto son insostenibles y que es necesaria una transformación de la seguridad social. Hasta aquí puede que haya el acuerdo total y las opiniones que siguen causen debate.

-Sin embargo, ante lo señalado en el apartado anterior, el camino de las cuentas individuales debe tomar otros parámetros de organización y desempeño distintos a los adoptados en las reformas del IMSs. Para subsanar las deficiencias apuntadas, el camino de la capitalización debe tomar formas más colectivas, buscar costos de administración menores, garantizar las inversiones y aplicarse a inversión productiva (Bonilla y Conte-Grand, 1999; Farell, 1999).

-Las opciones de solución deben revisar el significado de una pensión y los derechos para adquirirla (Valencia, 1999b). Entre estos conceptos están el significado de una pensión, la edad al retiro, la calificación de una invalidez, la antigüedad mínima requerida, los montos de la pensión y el tratamiento fiscal (Bonilla y Conte-Grand, 
1999). En esta tarea deben revaluarse los sistemas de privilegio y todas las implicaciones políticas y sindicales que conlleva.

-En su mayoría las muchas y distintas instituciones de seguridad social del país aún no han realizado modificaciones, aunque sí han iniciado valuaciones actuariales y consideraciones en ese sentido. Estas instituciones tienen tal variedad de situaciones (Farell, 1998) que las reformas que lleven a cabo no deberán ser uniformes, sino seguir caminos propios.

-Para satisfacer las necesidades de investigación que pueden proporcionar elementos de valuación, planeación y negociación, deben realizarse ejercicios de evaluación actuarial y de modelaje econométrico que permitan la construcción de escenarios de prospectiva bajo distintas alternativas de proyección que consideren la diversidad de sistemas existentes.

-No debe olvidarse que las pensiones son sólo una parte de la problemática de la seguridad social. Otros elementos esenciales para el bienestar vienen con la prevención económica personal, el cuidado de la salud y la construcción de relaciones familiares, en una interrelación evidentemente necesaria. El comportamiento que se observe en estos renglones habrá de impactar la necesidad y uso de las pensiones. Así resulta indispensable el abordaje integral e interdisciplinario sobre la salud y el bienestar, incluyendo a las pensiones en sus distintas modalidades. Asimismo, debe considerarse que la propia seguridad social y los sistemas de pensiones son parte de la dinámica social y económica de la nación.

-Un punto importante en las condiciones demográficas de México resulta de las estructuras de población que se esperan en las próximas décadas. Estas estructuras indican una disminución en los grupos dependientes de niños y adolescentes que sobrepasa el aumento de los dependientes en edades envejecidas, en un aparente mejoramiento de los niveles de dependencia. En todo caso se trata de incrementos de los grupos de población en edades productivas y capaces de otorgar cuidados, lo cual brinda una oportunidad, pero siempre y cuando se pueda lograr una recomposición económica y social de toda la nación que permitiera otorgar trabajo pleno y formal en las décadas por venir (Ham, 1995). Cómo puede la seguridad social ser parte crucial de esta recomposición es una tarea aún por emprenderse.

-Finalmente, también deben tomarse en cuenta las experiencias que se realizan en otras sociedades parecidas a la nuestra. Resulta relevante, en primer lugar, que el desempeño de las reformas chilenas 
está lejos de mantener el ritmo elevado y a largo plazo que se requiere y que supuestamente se lograría en beneficio de los pensionados futuros, pero que ya muestra deficiencias (Ruiz-Tagle, 1996). Asimismo, en los debates y críticas sobre las reformas en otros países sobresale que en Brasil se ha cancelado la reforma y se ha optado por establecer mejores controles sobre la concesión de beneficios, para que el gobierno federal cubra las pensiones en curso de pago; en Costa Rica se ha implantado la cuenta individual pero como esquema voluntario, el cual tiene como costo un tope máximo de $10 \%$ de las utilidades reales; en Uruguay un referéndum propone que en los salarios menores no se aplique este sistema, en los intermedios sea volun tario y sólo se aplique en los ingresos altos (Soto, 1999).

\section{Bibliografía}

Arancibia Córdova, Juan y Berenice P. Ramírez (1999), "Tendencias y perspectivas del proceso de reforma de la seguridad social en América Latina”, en Berenice P. Ramírez, La seguridad social: reformas y retos, México, IIE, UNAM/Porrúa.

Bonilla, Alejandro y Alfredo H. Conte-Grand (1999), "Las reformas de los regímenes de pensiones en América Latina", en OIT, La reforma estructural de pensiones en América Latina: dos décadas de reforma. Ginebra, Oficina Internacional del Trabajo.

Briseño, Alberto (1999), El Financiero, México, 3 de mayo.

Conapo (1999), Proyecciones de la población de México, 1995-2050, México, Consejo Nacional de Población.

DOF (1995), "Ley del Seguro Social", Diario Oficial de la Federación, México, 21 de diciembre.

Farell, Rosa M. (1998), Informe sobre las pensiones estatales, México, Taller sobre Pensiones Estatales, junio.

__ (1999), "Evaluación y perspectivas de la seguridad social", en Conapo, Envejecimiento demográfico de México: retos y perspectivas, México, Consejo Nacional de Población.

Forrester, Viviane (1996), L'horreuréconomique, París, Librairie Artème Fayard.

Guhan, S. (1994), "Social Security Options for Developing Countries", International Labour Review, vol. 133, núm. 1.

Ham-Chande, Roberto (1995), Ageing in Mexico: Another Challengeffor a Middle Income Country, Valletta, Malta, CICRED-INIA.

Hazas, Alejandro (1996), "Futuro del sistema pensionario de los trabajadores sujetos a la nueva ley del IMSs", en M. L. Mussot, Alternativas de reforma de la seguridad social, México, UAM/Fundación Ebert. 
Heller, Peter S. (1998), "Rethinking Public Pension Reform Iniciatives", trabajo presentado en el APEC Regional Forum on Pension Fund Reforms, Cancún, México.

IMSS (1985), 40 Años de historia del IMSS, México, Instituto Mexicano del Seguro Social.

(1995), Diagnóstico, México, Instituto Mexicano del Seguro Social.

(1997), Memoria estadística, 1996, México, Instituto Mexicano del Seguro Social.

ISSSTE (1997), Anuario estadístico, 1996, México, Instituto de Servicios y Seguridad Social para los Trabajadores del Estado.

Laurell, Cristina (1996), "La reforma del IMSS: pieza clave del proyecto neoliberal", El Cotidiano, núm. 78.

Mesa-Lago, Carmelo (1986), La crisis de la seguridad social y la atención a la salud. Experiencias recientes y lecciones latinoamericanas, México, Fondo de Cultura Económica.

OIT (1984), Introducción a la seguridad social, Ginebra, Oficina Internacional del Trabajo.

Partida, Virgilio (1998), La dinámica poblacionaly su impacto en el sistema de pensiones, México, Taller sobre Pensiones Estatales, junio.

Pedrero, Mercedes (1998), "Situación económica en la tercera edad", ponencia presentada en el Taller Interdisciplinario sobre Conceptos y Metodología en el Estudio del Envejecimiento, Sociedad Mexicana de Demografía.

Ramírez, Berenice y Juan Arancibia (1999), "A manera de conclusión”, en Berenice P. Ramírez, La seguridad social: reformas y retos, México, IIEUNAM/Porrúa.

Ruiz-Tagle, Jaime (1996), "El nuevo sistema de pensiones en Chile: una evaluación preliminar", Comercio Exterior, vol. 46, núm. 9.

Salas, J. L. (1998), "Evaluación actuarial a la reforma de la seguridad social", ponencia presentada en el Taller sobre las Reformas a la Seguridad Social: Análisis de las Posibilidades Económicas y Sociales del Nuevo Sistema de Pensiones, Cuernavaca, Somede, mayo.

Sandoval-Bustos, Maritza (1998), "Análisis del efecto de la reforma al sistema de pensiones del IMSS sobre la inversión privada", tesis de maestría en Economía, Tijuana, El Colegio de la Frontera Norte.

Soto, Carlos (1992a), "La seguridad social en México", en Sistemas de pensiones en América Latina y el Caribe: diagnóstico y alternativas de reforma, Chile, CEPAL.

(1992b) "Nota informativa sobre la situación actuarial del IMSS" (mimeo.). - (1999), "Reformas a los regímenes de pensiones en América Latina", ponencia presentada en el IV Congreso de Actuarios a Nivel Internacional, Girardot, Colombia.

Uthoff, Andras (1997), "Reformas a los sistemas de pensiones, mercado de capitales y ahorro", Revista de la CEPAL, diciembre. 
Valencia, A. (1999a), "El valor de los pasivos contingentes", en Conapo, Envejecimiento demográfico de México: retos y perspectivas, México, Consejo Nacional de Población.

- (1999b), Pensiones de la APF y su cobertura a la población de adultos mayores, México, Taller sobre Envejecimiento Demográfico, Trabajo y Previsión Social, STPS, julio.

Wallace, Paul (1999), Agequake, Londres, Nicholas Brealey Publishing Limited. 